\title{
A cohort study of infant feeding practices in city, suburban and rural areas in Zhejiang Province, PR China Liqian Qiu ${ }^{\dagger 1,2}$, Yun Zhao ${ }^{\dagger 2}$, Colin W Binns*2, Andy H Lee ${ }^{\dagger 2}$ and Xing Xie ${ }^{\dagger 1}$
}

Address: ${ }^{1}$ Women's Hospital, School of Medicine, Zhejiang University, PR China and ${ }^{2}$ School of Public Health, Curtin University, WA, Australia Email: Liqian Qiu - qiulq@zju.edu.cn; Yun Zhao - y.zhao@curtin.edu.au; Colin W Binns* - c.binns@curtin.edu.au; Andy H Lee - Andy.Lee@curtin.edu.au; Xing Xie - xinxie@zju.edu.cn

* Corresponding author †Equal contributors

Published: 3 March 2008

International Breastfeeding Journal 2008, 3:4 doi:10.1 186/1746-4358-3-4
Received: 4 October 2007

Accepted: 3 March 2008

This article is available from: http://www.internationalbreastfeedingjournal.com/content/3/I/4

(C) 2008 Qiu et al; licensee BioMed Central Ltd.

This is an Open Access article distributed under the terms of the Creative Commons Attribution License (http://creativecommons.org/licenses/by/2.0), which permits unrestricted use, distribution, and reproduction in any medium, provided the original work is properly cited.

\begin{abstract}
Background: Breastfeeding is the basis for appropriate nutrition for infants and is strongly supported by the Ministry of Health in China. However, there are differences in infant feeding practices in different areas of the country. The aim of this study was to compare the infant feeding practices and the prevalence of determinants of initiation and continuing to breastfeed until six months of age in city, suburb and rural areas in Zhejiang Province, PRC.
\end{abstract}

Methods: A longitudinal cohort study of infant feeding practices was undertaken in city, suburban and rural areas in 2004/2005. Mothers were recruited and interviewed before discharge from hospitals. A total of 1520 mothers were recruited into the study. Follow-up interviews were administered at I, 3 and 6 months after birth to obtain details of infant feeding practices.

Results: 'Any breastfeeding' rates were high before discharge at all three locations, $96.5 \%$ in city, $96.8 \%$ in suburb and $97.4 \%$ in the rural area. The 'exclusive breastfeeding' rates in the city, suburban and rural areas before discharge were $38.0 \%, 63.4 \%$ and $61.0 \%$ respectively. By sixth months the 'any breastfeeding' rates had declined to $62.8 \%, 76.9 \%$ and $83.6 \%$ and the 'exclusive breastfeeding' rates had fallen to $0.2 \%, 0.5 \%$ and $7.2 \%$ in city, suburb and rural areas respectively. There were differences in feeding practices between the three locations, including the use of prelacteal feeds and the introduction of supplementary feeds.

Conclusion: Mothers who lived in the city were least likely to be 'exclusive breastfeeding' at discharge. At six months the city infants also had lower rates of 'any breastfeeding' and 'exclusive breastfeeding'.

\section{Background}

The Ministry of Health in China has recognized the importance of breastfeeding in infant nutrition and recommends exclusive breastfeeding for the first six months of life [1]. Like other developing countries, China was influenced in the $19^{\text {th }}$ and $20^{\text {th }}$ centuries by western practices and its traditional pattern of home-based delivery became hospital based. Mothers were often separated from their infants in hospitals and infant formula became more readily available. These factors and the improving economy led to a decline in the breastfeeding rate. In Shanghai, one of China's largest cities, a large cross-sectional study was conducted in 1980, supported by the World Health Organization (WHO) which included a 
total of 3845 mothers recruited from the city and suburbs [2]. The data from this study showed the 'any breastfeeding' rate had declined to $24.8 \%$ in the city and $77.0 \%$ in the suburbs for 0-6 month old babies [2]. This study was technically supported by WHO and used the period prevalence method of recording breastfeeding rates recommended by WHO [3]. In the following years further surveys revealed similar trends in other regions of the country. In 1983 a national cross-sectional survey of 111,348 infants aged 0-6 months found that the 'any breastfeeding' rate was $49.3 \%$ in the city and $75.1 \%$ in rural areas [4]. The decline in breastfeeding rates was a challenge for China as she sought to achieve the goals set at the International Child Survival Conference in 1990 which were endorsed by the Chinese Premier [1].

The Chinese government strongly supported the international goals as a way of improving the nutritional status of her children and in the early 1990s the Ministry of Public Health in China began promoting breastfeeding on a large scale across the nation. Several projects were launched to encourage breastfeeding and promote its benefits to parents and health professionals. These projects included initiating scientific research on the promotion of breastfeeding, promoting 'rooming in' and increasing health education about the benefits of breastfeeding. The State Council passed regulations to extend maternity leave from six weeks to three months to support breastfeeding. Employers were instructed to ensure that mothers had sufficient time for nursing if continuing to breastfeed and nursing rooms were required to be provided in work units. At the same time the first draft of the "National Program for the Promotion of Breast Feeding in China" was circulated [1]. The promotion of the "Baby Friendly Hospital Initiative" was commenced in China and initially a few maternity hospitals were accredited, followed by some of the larger integrated hospitals. Breastfeeding rates began to rise in the 1990s as "Baby Friendly accreditation" promoted by the Ministry of Health, spread across the country.

Zhejiang Province is located on the east coast of China, south of Shanghai and in 2006 had a population of 49 million. Since the Chinese economic reforms began 30 years ago, Zhejiang Province has developed its economy, education and health care systems and has become one of the most prosperous regions in the nation. The capital of Zhejiang Province is Hangzhou, a city of four million, first made famous when Marco Polo was appointed its governor in the $12^{\text {th }}$ century. Hangzhou has become the centre of large information technology and electronics industries (Figure 1). Like other large cities in China, in Hangzhou, the breastfeeding rate declined with the introduction of western patterns of obstetric services. However, after the nationwide launch of the Baby Friendly Hospital Initia-

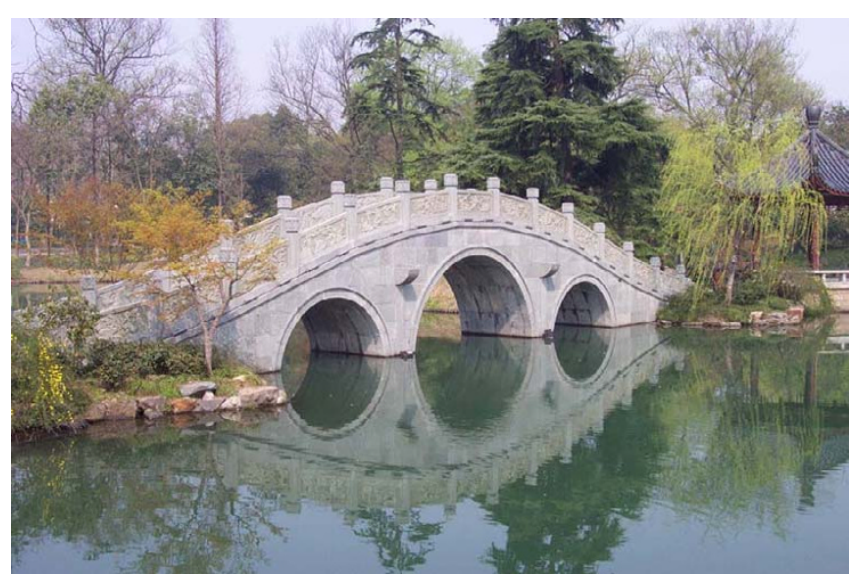

Figure I

West Lake, Hangzhou.

tive in China, more than $85 \%$ of maternity and integrated hospitals in Zhejiang Province changed their existing systems to become "Baby Friendly" by meeting the WHO/ UNICEF criteria. However, some barriers to breastfeeding still exist in hospitals and in the community and exclusive breastfeeding of infants up to six months of age is uncommon in Zhejiang Province. The rate is much lower than Chinese and international targets. This has important implications for the health of children of the province.

Because breastfeeding statistics are incomplete and there is little information about the reasons for breastfeeding and 'not breastfeeding', a longitudinal cohort study was commenced in Zhejiang Province. The aim of the study was to determine the prevalence of breastfeeding and prevalence of determinants of initiation and continuing to breastfeed until six months of age in city, suburban and rural areas of Zhejiang Province.

\section{Methods}

A longitudinal cohort study of infant feeding practices was undertaken in three locations in Zhejiang Province, in the capital city (Hangzhou), in a suburban location (Fuyang) which is located $50 \mathrm{~km}$ to the southwest of Hangzhou and in a mountainous rural area a further $300 \mathrm{~km}$ to the southwest. All the mothers in the study were recruited while in hospital during the period October 2004 to December 2005. The first interview was undertaken by a nurse or women's health worker before discharge from hospital and follow up interviews were held at one month, three months and six months postpartum. The first interview was always undertaken in person and most of the followup interviews (92\%) were undertaken by telephone. In the few instances where mothers could not be reached by telephone, the follow-up interviews were completed at the routine examinations in the community child care clinics. 
A total of 1520 mothers were recruited from four hospitals; the Women's Hospital, School of Medicine, Zhejiang University in the city, Fu-yang Maternal and Child Hospital in the suburban location and Jin-Yun People's Hospital and Li-Shui Maternal and Child Hospital in the rural area. Each of these hospitals is typical of the health care facilities in the area they are located. The inclusion criteria for the study were that the mother had delivered a live child, the mother and neonate did not have serious diseases and that she was resident in the service area of that hospital. In Hangzhou almost one half of the births were not of local residents and were excluded from the study. However, in the suburban and rural areas almost all mothers were local residents and were eligible to participate. In order to be able to manage the number being interviewed on any one day, selection was made using a series of random numbers. The response rate was high and $98.0 \%$ of mothers (1520 out of 1551) agreed to participate in the study.

The questionnaires were based on those developed by Scott, Binns, Xu and Duong that have been used extensively in breastfeeding cohort studies in Australia, Xinjiang, China and Vietnam [5-7]. The questionnaires were designed to identify the feeding method and to collect information on factors associated with breastfeeding. After translation the questionnaires were pilot tested in city and rural areas and were modified for the Zhejiang language and culture.

The project was approved by the Research Administration Section of the Women's Hospital, School of Medicine, Zhejiang University and the Human Research Ethics Committee of Curtin University, Australia. The purpose of the study was explained to the mothers and assurance was given that all information would be kept confidential. Any mother had the right to withdraw from the study at anytime without prejudice. After the purpose of the study had been explained to the mothers, they were given a consent letter to sign. The questionnaire and the interviewing nurses used standard terminology and the local dialect to ensure mothers' understanding.

All data analyses were carried out using the Statistical Package for the Social Sciences, release 14.0 (SPSS Inc.). Descriptive statistics and cross-tabulations were generated for demographic factors, life tables were used for breastfeeding rates and binary logistic regression was used to calculate odds ratio of infant formula use.

The definitions of breastfeeding used in this paper were based on standard definitions and were the same as those used in Xu's study in Xinjiang Province, in the west of China [6]:
- Any breastfeeding: The infant receives breastmilk (direct from the breast or expressed) with or without any other drink, formula or other infant food.

- Exclusive breastfeeding: Breastfeeding while giving no other food or liquid, not even water, with the exception of drops or syrups consisting of vitamins, mineral supplements or medicine.

Prelacteal feeds are defined as any feeds given before the onset of lactogenesis II, which is the onset of copious lactation that occurs within four days of birth [8].

\section{Results}

A total of 1520 mothers were recruited into the study, $42.0 \%$ from the city, $22.8 \%$ from the suburban area and $35.2 \%$ from the rural area. Almost all mothers were married $(99.9 \%)$, and most belonged to the Han ethnic group $(97.5 \%)$. The details of the study sample and the prevalence of the major demographic variables in the city, suburban and rural areas are shown in Table 1.

Generally the mothers from the city had higher levels of education, a higher proportion of office employment and higher family income compared to the mothers in the suburban and rural areas. Mothers from the city usually were older and only $12.0 \%$ of mothers in the city had their babies before the age of 25 years compared to $35.7 \%$ in the suburban and $30.6 \%$ in the rural locations. Mothers in the city and suburban area were more likely to give birth by caesarean section: $75.7 \%$ in the city and $74.1 \%$ in the suburban area, compared to $52.8 \%$ in the rural area.

The proportions of mothers who were breastfeeding before hospital discharge from the three locations are shown in Table 2. More than $95 \%$ of mothers in each location initiated breastfeeding, but the exclusive breastfeeding rate was much lower in the city. A high proportion of mothers used prelacteal feeds on at least one occasion before discharge as previously reported [9].

The breastfeeding rates in the three locations were analysed using life table analysis and are detailed in Table 3, Figures 2 and 3. Figure 2 shows the 'any breastfeeding' rates and Figure 3 the 'exclusive breastfeeding' rates for the three locations in Zhejiang Province. While the majority of mothers were continuing to breastfeed at six months, only a few infants were exclusively breastfed at this age, especially in the city and suburbs.

The exclusive breastfeeding rate was lower than the national target ( $80 \%$ till four months of age) in all locations and at all ages. A lower proportion of mothers in the city $(38.0 \%)$ were exclusively breastfeeding compared to 
Table I: Demographic details of mothers' in the city, suburb and rural areas, Zhejiang Province, People's Republic of China, 2004-2005 $(n=1520)$

\begin{tabular}{|c|c|c|c|c|c|c|c|c|}
\hline \multirow[t]{2}{*}{ Variable } & \multicolumn{2}{|c|}{ City } & \multicolumn{2}{|c|}{ Suburb } & \multicolumn{2}{|c|}{ Rural } & \multicolumn{2}{|c|}{ Total } \\
\hline & $\mathbf{n}$ & $\%$ & $\mathbf{n}$ & $\%$ & $\mathbf{n}$ & $\%$ & $\mathbf{n}$ & $\%$ \\
\hline Residence & 638 & 42.0 & 347 & 22.8 & 535 & 35.2 & 1520 & 100.0 \\
\hline \multicolumn{9}{|c|}{ Maternal Age (years)* } \\
\hline$<25$ & 77 & 12.0 & 124 & 35.7 & 158 & 30.6 & 358 & 23.9 \\
\hline $25-29$ & 383 & 60.2 & 165 & 47.6 & 255 & 49.2 & 800 & 53.5 \\
\hline $30-34$ & $15 \mid$ & 23.9 & 51 & 14.7 & 80 & 15.5 & 282 & 18.9 \\
\hline$\geq 35$ & 25 & 3.9 & 7 & 2.0 & 24 & 4.7 & 56 & 3.7 \\
\hline Missing & 5 & & 0 & & 19 & & 24 & \\
\hline \multicolumn{9}{|c|}{ Maternal education (years)* } \\
\hline$\leq 9$ & 57 & 9.0 & 206 & 59.4 & 282 & 53.1 & 544 & 36.0 \\
\hline $10-12$ & 172 & 26.9 & 78 & 22.5 & 121 & 22.9 & 370 & 24.5 \\
\hline$>12$ & 407 & 64.1 & 63 & 18.2 & 127 & 24.0 & 597 & 39.5 \\
\hline Missing & 3 & & & & 6 & & 9 & \\
\hline \multicolumn{9}{|c|}{ Maternal employment* } \\
\hline Labour job & 67 & 10.5 & 188 & 55.1 & 238 & 47.1 & 492 & 33.3 \\
\hline Office job & 494 & 78.4 & 90 & 26.4 & 179 & 35.4 & 763 & 51.7 \\
\hline Not employed & 70 & 11.1 & 63 & 18.5 & 89 & 17.4 & 221 & 15.0 \\
\hline Missing & 8 & & 6 & & 30 & & 44 & \\
\hline \multicolumn{9}{|c|}{ Gestation (weeks)* } \\
\hline$<37$ & 30 & 4.7 & 7 & 2.0 & 12 & 2.4 & 49 & 3.3 \\
\hline$\geq 37$ & 605 & 95.3 & 340 & 98.0 & 499 & 97.6 & 1443 & 96.7 \\
\hline Missing & 3 & & 0 & & 25 & & 28 & \\
\hline \multicolumn{9}{|l|}{ Birth weight (g) } \\
\hline$<2500$ & 9 & 1.4 & 7 & 2.0 & 11 & 2.1 & 27 & 1.8 \\
\hline $2500-3999$ & 583 & 91.8 & 310 & 89.3 & 489 & 93.3 & 1382 & 91.8 \\
\hline$\geq 4000$ & 43 & 6.8 & 30 & 8.6 & 24 & 4.6 & 97 & 6.4 \\
\hline Missing & 3 & & 0 & & 11 & & 14 & \\
\hline \multicolumn{9}{|c|}{ Delivery method* } \\
\hline Vaginal & 155 & 24.3 & 90 & 25.9 & 252 & 47.2 & 495 & 32.7 \\
\hline Caesarean & 481 & 75.7 & 257 & 74.1 & 281 & 52.8 & 1019 & 67.3 \\
\hline Missing & 3 & & 0 & & 3 & & 6 & \\
\hline \multicolumn{9}{|l|}{ Birth order* } \\
\hline I & 615 & 96.9 & 290 & 84.1 & 442 & 83.4 & 1347 & 89.2 \\
\hline$\geq 2$ & 20 & 3.1 & 55 & 15.9 & 89 & 16.6 & 163 & 10.8 \\
\hline Missing & 3 & & 2 & & 5 & & 10 & \\
\hline \multicolumn{9}{|l|}{ Baby's gender } \\
\hline Male & 347 & 54.2 & 162 & 47.4 & 260 & 49.7 & 767 & 51.1 \\
\hline Female & 291 & 45.8 & 180 & 52.6 & 263 & 50.3 & 734 & 48.9 \\
\hline Missing & 2 & & 5 & & 12 & & 19 & \\
\hline \multicolumn{9}{|c|}{ Monthly family income* (RMB) } \\
\hline$\leq 1500$ & 6 & 1.0 & 51 & 15.0 & 158 & 31.3 & 215 & 14.6 \\
\hline$|50|-3000$ & 75 & 11.9 & 145 & 42.8 & 182 & 35.9 & 401 & 27.2 \\
\hline $3001-5000$ & 227 & 35.7 & 97 & 28.6 & 129 & 25.6 & 451 & 30.6 \\
\hline$>5000$ & 326 & 51.5 & 46 & 13.6 & 36 & 7.1 & 407 & 27.6 \\
\hline Missing & 7 & & 8 & & 31 & & 46 & \\
\hline
\end{tabular}

$*_{\mathrm{p}}<0.05$

the suburban $(63.4 \%)$ and rural $(61.0 \%)$ areas on discharge.

The prevalence data were converted to period prevalence, to allow comparison with WHO statistics and are shown in Table 4.
Supplementary feeding with infant formula and other complementary foods is quite common in China. The prevalence of formula feeding at the different locations at three and six months of age is described in Table 5.

In Table 5, the crude odds ratios and confidence intervals were calculated using binary logistic regression analysis. 
Table 2: 'Any breastfeeding', 'exclusive breastfeeding' and prelacteal feeding rates at discharge in Zhejiang Province, PR China, 20042005

\begin{tabular}{|c|c|c|c|c|c|c|}
\hline & \multicolumn{2}{|c|}{ 'Any breastfeeding' } & \multicolumn{2}{|c|}{ 'Exclusive breastfeeding' } & \multicolumn{2}{|c|}{ Prelacteal feeding } \\
\hline & $\mathbf{n}$ & $\%$ & $\mathbf{n}$ & $\%$ & $\mathbf{n}$ & $\%$ \\
\hline City & 613 & 96.5 & 233 & 38.0 & 380 & 62.0 \\
\hline Suburb & 336 & 96.8 & 213 & 63.4 & 123 & 36.6 \\
\hline Rural & 518 & 97.4 & 316 & 61.0 & 202 & 39.0 \\
\hline
\end{tabular}

Compared with mothers living in rural areas, mothers who lived in the city were more likely to use formula by the third month after birth (OR: 2.10, 95\%CI: 1.61, 2.73). Mothers who lived in the suburban area were less likely to be using formula feeding (OR: 0.67, 95\%CI: 0.50, 0.88).

By the sixth month very few mothers in both city and suburban area were exclusively breastfeeding $(0.2 \%$ and $0.5 \%$ respectively), but in the rural area the rate was higher where $7.2 \%$ of mothers were still exclusively breastfeeding. The infant feeding practices of the mothers from the city and suburban area were similar and when these two groups were combined and compared to the rural group the contrast at six months was even stronger (Crude OR:11.38, 95\%CI: 5.13, 25.24).

\section{Discussion}

The economy of Zhejiang Province has grown rapidly in recent years, particularly since the beginning of the $21^{\text {st }}$ century and the per capita GDP in Hangzhou was \$6,700 in 2006. The rise in living standards accompanying the economic growth has led to a demand for improved health care and in particular, the application of advanced medical technologies. Infant feeding practices are another part of the culture which has been influenced by economic development. There is now widespread promotion

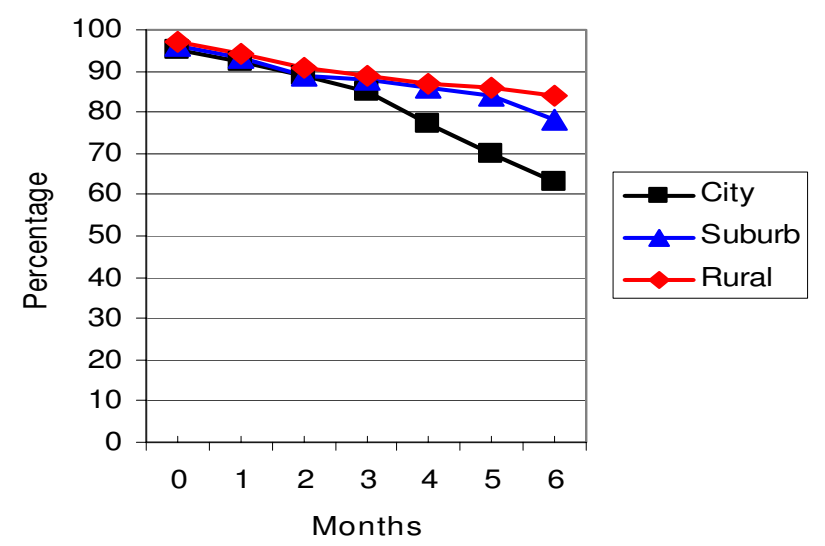

Figure 2

'Any breastfeeding' rates Zhejiang Province. of infant formula and mothers are fascinated by the prospect of a high-technology product which promises much for their infants. On the other hand, cultural beliefs are still strong and most mothers commence breastfeeding, but they tend to combine this with infant formula in the early months of their infants' lives.

Chinese society has changed rapidly and in the "hightech" city of Hangzhou it is now very common for women to pursue higher education and to marry later in life. The women of Hangzhou are highly educated and in our study $64.1 \%$ of the women had post secondary education compared with the rates of $18.2 \%$ in the suburban area and $24.0 \%$ in the rural area. Almost half of the mothers (45.5\%) considered for recruitment into the study were not native residents of the city, compared to the suburban $(18.4 \%)$ and the rural areas (3.7\%). This reflects the dynamic nature of the Hangzhou population resulting from the rapid economic development.

There have been rapid changes in medical practice in the past decades and this is reflected in changes in birthing methods. Overall in the study, two thirds of mothers gave birth by caesarean section. The rates in the city and suburban and rural areas were $75.7 \%, 74 \%$, and $52.7 \%$ respectively. After the Baby Friendly Hospital Initiative (BFHI) was promoted in China in the 1990s, the majority of hos-

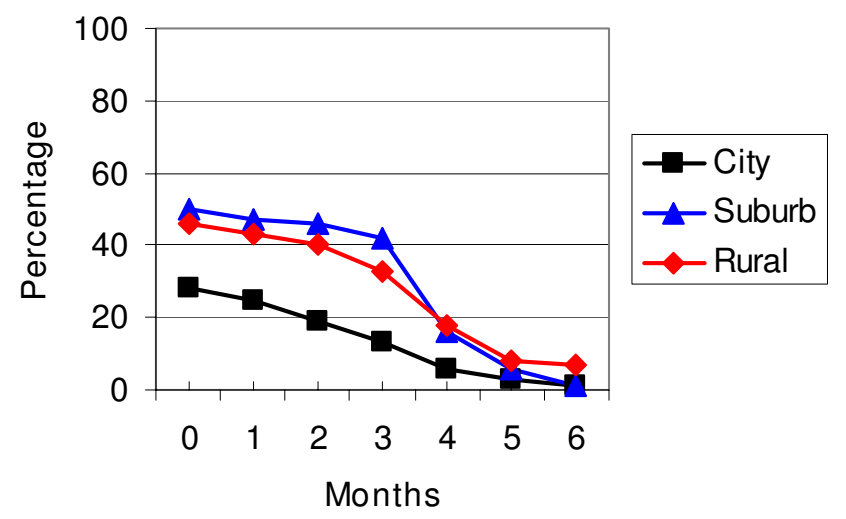

Figure 3

'Exclusive breastfeeding' rates Zhejiang Province. 
Table 3: 'Any breastfeeding' and 'exclusive breastfeeding' rates from I-6 months in city, suburban and rural Zhejiang Province, PR China

\begin{tabular}{|c|c|c|c|c|c|c|c|}
\hline \multirow[t]{2}{*}{ Location } & \multirow[t]{2}{*}{ Age months } & \multicolumn{3}{|c|}{ 'Any breastfeeding' } & \multicolumn{3}{|c|}{ 'Exclusive breastfeeding' } \\
\hline & & $\mathbf{n}$ & $\%$ & $95 \% \mathrm{Cl}$ & $\mathbf{n}$ & $\%$ & $95 \% \mathrm{Cl}$ \\
\hline \multirow[t]{6}{*}{ City } & $\mathrm{I} \sim$ & 590 & 91.7 & $89.4,93.9$ & 166 & 24.4 & $21.0,27.7$ \\
\hline & $2 \sim$ & 380 & 88.6 & $85.8,91.3$ & 119 & 18.8 & $15.6,22.0$ \\
\hline & $3 \sim$ & 367 & 84.0 & $80.7,87.2$ & 92 & 13.7 & $10.8,16.6$ \\
\hline & $4 \sim$ & 347 & 76.2 & $72.3,80.1$ & 67 & 5.5 & $3.5,7.5$ \\
\hline & $5 \sim$ & 315 & 69.7 & $65.4,74.0$ & 27 & 1.8 & $0.7,3.0$ \\
\hline & $6 \sim$ & 288 & 62.8 & $57.7,67.9$ & 9 & 0.2 & NA \\
\hline \multirow[t]{6}{*}{ Suburb } & $\mathrm{I} \sim$ & 325 & 90.4 & $87.3,93.5$ & 174 & 47.0 & $41.7,52.3$ \\
\hline & $2 \sim$ & 311 & 88.4 & $85.0,91.8$ & 161 & 45.8 & $40.6,51.1$ \\
\hline & $3 \sim$ & 304 & 86.6 & $83.0,90.2$ & 157 & 41.7 & $36.5,46.9$ \\
\hline & $4 \sim$ & 290 & 84.5 & $80.7,88.3$ & 140 & 16.1 & $12.2,20.0$ \\
\hline & $5 \sim$ & 283 & 82.7 & $78.7,86.7$ & 54 & 4.5 & $2.3,6.7$ \\
\hline & $6 \sim$ & 277 & 76.9 & $71.8,82.0$ & 15 & 0.5 & NA \\
\hline \multirow[t]{6}{*}{ Rural } & $\mathrm{I} \sim$ & 499 & 93.5 & $91.4,95.6$ & 201 & 42.7 & $38.1,47.3$ \\
\hline & $2 \sim$ & 486 & 90.6 & $88.1,93.1$ & 189 & 39.8 & $35.2,44.3$ \\
\hline & $3 \sim$ & $47 I$ & 89.0 & $86.4,91.7$ & 176 & 33.1 & $28.8,37.5$ \\
\hline & $4 \sim$ & 456 & 86.9 & $84.0,89.8$ & 143 & 18.3 & $|4.7,2| .9$ \\
\hline & $5 \sim$ & 445 & 85.1 & $82.1,88.2$ & 79 & 8.1 & $5.5,10.7$ \\
\hline & $6 \sim$ & 436 & 83.6 & $80.2,87.0$ & 35 & 7.2 & $4.7,9.8$ \\
\hline
\end{tabular}

pitals in the Province reformed their obstetric practices. The hospital environment became friendly towards babies, as the Ten Steps to Successful Breastfeeding were required in all hospitals and maternal and child health centres. These included 'rooming in', early skin contact, early initiation of breastfeeding and the encouragement of mothers to breastfeed by the doctors and nurses in the obstetric departments. The Bureau of Public Health of Zhejiang Province was responsible for providing a team to regularly evaluate the Baby Friendly hospitals in the Province and to maintain the quality of the BFHI. In this atmosphere of encouragement, breastfeeding initiation rates improved. Almost all mothers understood the benefits of breastfeeding from antenatal classes, from postnatal education or from the media.

In our study more than $96 \%$ of mothers were breastfeeding their infants at discharge from hospital. The initiation of breastfeeding has returned to higher levels in the past two decades, and the rates of breastfeeding initiation in Zhejiang are now higher than reported from other provinces in China. A study of infant feeding in Xinjiang Uygur

Table 4: The period prevalence (0-6 months) of 'any' and 'exclusive' breastfeeding in Zhejiang Province, PR China

\begin{tabular}{lccc}
\hline & n & $\begin{array}{c}\text { 'Any breastfeeding' } \\
\%\end{array}$ & $\begin{array}{c}\text { 'Exclusive breastfeeding' } \\
\%\end{array}$ \\
\hline City & 406 & 81.1 & 13.0 \\
Suburb & 342 & 86.0 & 29.5 \\
Rural & 530 & 89.2 & 27.8 \\
\hline
\end{tabular}

Autonomous China found an 'any breastfeeding' rate of 92.2\% at discharge [6]. The 'Beijing and Four Provinces Study' found that the 'ever breastfed' rate was $90.1 \%$ in China in 2002 [10]. This rate was similar to an Australia infant feeding study from West Australia where 93.5\% of mothers were breastfeeding at discharge from hospital [11].

The 'any breastfeeding' rate for the city is higher than the rate reported for Shanghai, the closest large city to Hangzhou in the 1980s [2]. This probably reflects a renewed interest in breastfeeding in China. However the situation for 'exclusive breastfeeding' is not as encouraging. The WHO reported the 0-6 months exclusive breastfeeding rate in China to be $51 \%$ [12]. Our results for 'exclusive breastfeeding' are far below this figure and may reflect different methodology. The WHO-UNICEF methodology relies on 24 hour recall of no foods or fluids apart from breast milk in the past 24 hours. China is a vast country with a huge population and another reason for the difference may be sample selection. The variation from our study suggests that larger and more widespread studies of breastfeeding are needed and they should preferably use the longitudinal methodology used in this study. Another factor influencing breastfeeding in the city is the economic pressure of the developing economy. In an ethnographic study of women in Beijing, Gottschang summarized the pressures on urban mothers: "global intervention in the form of the WHO-UNICEF sponsored Baby Friendly Hospital Initiative promotes breastfeeding as a women's duty at the same time that market forces counter this message" [13] (p. 64). 
Table 5: The prevalence of infant formula or other complementary food use in city, suburb and rural areas Zhejiang Province, PR China

\begin{tabular}{|c|c|c|c|c|c|c|c|c|}
\hline & \multicolumn{4}{|c|}{$\begin{array}{l}\text { Using infant formula or other complementary food at } 3 \\
\text { months }\end{array}$} & \multicolumn{4}{|c|}{$\begin{array}{c}\text { Using infant formula or other complementary food at } 6 \\
\text { months }\end{array}$} \\
\hline & $n$ & $\%$ & OR* & $95 \% \mathrm{Cl}$ & $\mathbf{n}$ & $\%$ & OR* & $95 \% \mathrm{Cl}$ \\
\hline Rural & 530 & 42.3 & I & & 469 & 89.8 & I & \\
\hline City & 406 & 32.7 & 2.10 & $1.61,2.73$ & $705 \#$ & 99.0 & 11.38 & $5.13,25.24$ \\
\hline Suburb & 342 & 60.6 & 0.67 & $0.50,0.88$ & & & & \\
\hline
\end{tabular}

*OR = crude odds ratio.

\#City and suburban data are combined to calculate the Odds Ratio at 6 months

Most mothers had an understanding of the need for breastfeeding at the beginning of their infant's life, but the exclusive breastfeeding rates after discharge from hospital declined rapidly. The exclusive breastfeeding rate was considerably lower than the national target of $80 \%$ of babies being exclusively breastfed until four months of age. In our study the exclusive breastfeeding rate at discharge was only one third $(38.0 \%)$ in the city and even in the suburban and the rural areas, the rates were $63.4 \%$ and $61.0 \%$ respectively. Prelacteal feeds were common in the initial days after birth, details of which have previously been reported [9]. The exclusive breastfeeding rate in Zhejiang was lower than that in Xinjiang Uygur Autonomous Region, China and in Vietnam, where the rates at discharge were $66.2 \%$ [6] and $83.6 \%$ respectively [7]. However the exclusive breastfeeding rate at six months in Xinjiang was similar to the rural area in this study.

At three months, one-half of the mothers were regularly giving their infants some infant formula. A common reason for giving formula or other complementary food is the belief that 'the more or the quicker the baby gained weight, the healthier the baby is'. In the Chinese culture, parents and grandparents are devoted to their children and with the 'one child policy' the centrality of the child in Chinese culture has increased in recent decades. Thus there is always cultural pressure to give the infant supplementary foods.

The breastfeeding rates found in this study are well below international and national targets. If the rates found in this study reflect national trends in China, it would mean that China is falling behind in its quest to meet the Millennium Goals. This suggests that further health promotion programs for breastfeeding and particularly for the extension of the period of exclusive breastfeeding are required. There needs to be continuing monitoring of the implementation of the Baby Friendly Hospital principles in the Province. Further research is needed into ways of increasing community support for breastfeeding and increasing the number of 'Baby Friendly' workplaces.
There are some limitations that need to be considered when interpreting the results of this study. The hospitals used were selected to be representative of their locations, but a larger probability sample would be required to be certain that the selected sample represented Zhejiang Province. A series of focus group discussions and/or indepth interviews with mothers and the extended families would help in understanding further details about breastfeeding problems and beliefs about infant growth and supplementary feeds.

\section{Conclusion}

This is the first longitudinal cohort study published on infant feeding practices in city, suburban and rural areas in Zhejiang province, an economically advanced area typical of eastern China. The overall 'any breastfeeding' rate was high before discharge at $96.5 \%$ in city, $96.8 \%$ in suburban and $97.4 \%$ in rural areas. The 'exclusive breastfeeding' rates in city, suburban and rural areas before discharge were $38.0 \%, 63.4 \%$ and $61.0 \%$. The exclusive

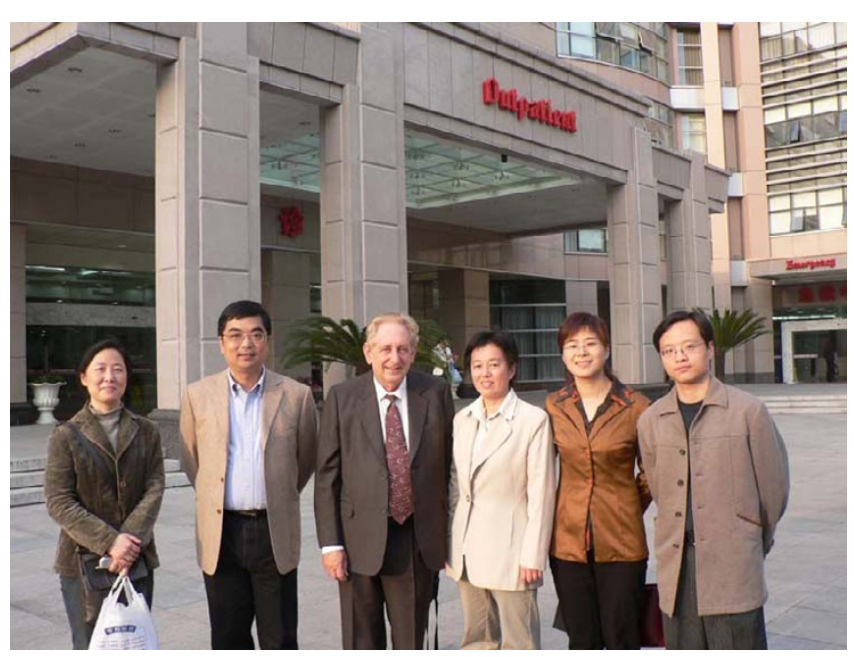

Figure 4

Authors (XX 2nd from left, CWB and LQ) and Hospital Staff, Women's Hospital, School of Medicine, Zhejiang University, PR China. 
breastfeeding rate was lower than the national target at discharge and also during the whole follow-up period until the infant was six months of age. The lower exclusive breastfeeding trend was most marked in the city. More studies are needed to find the detailed reasons related to the low rates of exclusive breastfeeding.

\section{Competing interests}

The authors declare that they have no competing interests.

\section{Authors' contributions}

All authors contributed to the study (see Figure 4). LQ designed the research, collected and analyzed data, drafted the manuscript. YZ analyzed data and revised the manuscript. CWB designed the research, drafted and revised the manuscript. AL analyzed data and revised the manuscript. XX designed the research, collected data and revised the manuscript.

\section{Acknowledgements}

We gratefully acknowledge the willing assistance given by all the mothers in our study, the hospital staff and nurses and health workers. This study would not have been possible without their assistance.

\section{References}

I. He J, Wang F: Baby friendly action in China. Department of Maternal and Child Health MoPH, P.R.China,. Beijing China; 1994.

2. Shanghai Children's Hospital Department of Biostatistics: Survey of breastfeeding status in 0-6 month babies Shanghai 198I. In The collection of breastfeeding research 1982-1994 Shanghai: Shanghai Medical University; 1995: I-4.

3. WHO: Infant and Young Child Feeding: A tool for assessing national practices, policies and programmes. Geneva: WHO; 2003.

4. Yang P, Zhan S, Ling J, Qiu C: Surveillance of breastfeeding status of 0-6 month babies in 20 provinces, P.R.China. In The collection of breastfeeding research 1982-1994 Shanghai: Shanghai Medical University; 1995:31-34.

5. Scott JA, Aitkin I, Binns CW, Aroni RA: Factors associated with the duration of breastfeeding amongst women in Perth, Australia. Acta Paediatr 1999, 88(4):416-42I.

6. Xu F, Binns C, Wu J, Yihan R, Zhao Y, Lee A: Infant feeding practices in Xinjiang Uygur Autonomous Region, People's Republic of China. Public Health Nutr 2007, I0(2): 198-202.

7. Duong DV, Binns CW, Lee $\mathrm{AH}$ : Breast-feeding initiation and exclusive breast-feeding in rural Vietnam. Public Health Nutr 2004, 7(2):795-799.

8. Neville MC, Morton J: Physiology and endocrine changes underlying human lactogenesis II. J Nutr 200 I, I 3 I:3005s-3008s.

9. Qiu L, Xie X, Lee A, Binns CW: Infants' first feeds in Hangzhou, PR China. Asia Pac J Clin Nutr 2007, I6(SuppI I):458-46I.

10. Zhang W, Hao B, Wang L: Breastfeeding in Beijing and four Provinces in China. Chinese Journal of Health Education 2004, 20:14-16.

11. Win NN, Binns CW, Zhao Y, Scott JA, Oddy WH: Breastfeeding duration in mothers who express breast milk: a cohort study. Int Breastfeed J 2006, I:28.

12. UNICEF: Progress for Children: a report card on nutrition number 4. New York 2006.

13. Gottschang SZ: Maternal bodies, breast-feeding, and consumer desire in urban China. Med Anthropol Q 2007, 2I(I):64-80.
Publish with Bio Med Central and every scientist can read your work free of charge

"BioMed Central will be the most significant development for disseminating the results of biomedical research in our lifetime. "

Sir Paul Nurse, Cancer Research UK

Your research papers will be:

- available free of charge to the entire biomedical community

- peer reviewed and published immediately upon acceptance

- cited in PubMed and archived on PubMed Central

- yours - you keep the copyright
BioMedcentral 